\title{
(C) OPEN ACCESS \\ Antithrombotic therapy after femoropopliteal artery stenting: 12-month results from Japan Postmarketing Surveillance
}

\author{
Osami Kawarada, ${ }_{1}^{1}$ Michikazu Nakai, ${ }^{2}$ Kunihiro Nishimura, ${ }^{2}$ Hideki Miwa, ${ }^{3}$ \\ Yusuke Iwasaki, ${ }^{4}$ Daitaro Kanno, ${ }^{5}$ Tatsuya Nakama, ${ }^{6}$ Yoshito Yamamoto, ${ }^{7}$ \\ Nobuhiko Ogata, ${ }^{8}$ Masato Nakamura, ${ }^{9}$ Satoshi Yasuda ${ }^{1}$
}

\begin{abstract}
- Additional material is published online only. To view please visit the journal online (http://dx.doi.org/10.1136/ heartasia-2018-011114).

For numbered affiliations see end of article.

\section{Correspondence to}

Osami Kawarada, Department of Cardiovascular Medicine, Ikuwakai Memorial Hospital, 3-20-29 Tatsumikita, Ikunoku, Osaka city, Osaka, 544-0004, Japan; osamikawarada@yahoo. co.jp
\end{abstract}

Received 26 August 2018 Revised 27 October 2018 Accepted 11 November 2018

\section{ABSTRACT}

Objective To investigate the effects of antithrombotic therapy on target lesion revascularisation (TLR) and major adverse cardiovascular and cerebrovascular events (MACCEs) at 12 months after femoropopliteal intervention with second-generation bare metal nitinol stents.

Methods A total of 277 lesions in 258 limbs of 248 patients with de novo atherosclerosis in the above-theknee femoropopliteal segment were analysed from the Japan multicentre postmarketing surveillance.

Results At discharge, dual antiplatelet therapy (DAPT) was prescribed in $68.5 \%$ and cilostazol in $30.2 \%$ of patients. At 12 months of follow-up, prescriptions of DAPT significantly $(p=0.0001)$ decreased to $51.2 \%$ and prescription of cilostazol remained unchanged $(p=0.592)$ at $28.0 \%$. Prescription of warfarin also remained unchanged $(14.5 \%$ at discharge, $13.3 \%$ at 12 months, $p=0.70)$. At 12 months, freedoms from TLR and MACCE were $89.4 \%$ and $89.7 \%$, respectively. In a multivariate Cox proportional hazards model, neither DAPT nor cilostazol at discharge was associated with both TLR and MACCE at 12 months. However, warfarin at discharge was only independently associated with TLR at 12 months. Kaplan-Meier estimates demonstrated that warfarin at discharge yielded a significantly $(p=0.013)$ lower freedom from TLR at 12 months than no warfarin at discharge. Freedom from TLR at 12 months by the Kaplan-Meier estimates was $77.8 \%$ (95\% Cl 59.0\% to $88.8 \%$ ) in patients with warfarin at discharge and $91.2 \%$ (95\% Cl 86.3\% to $94.3 \%$ ) in those without warfarin at discharge.

Conclusions Clinical benefits of DAPT or cilostazol might be small in terms of TLR and MACCE at 12 months. Anticoagulation with warfarin at discharge might increase TLR at 12 months.

\section{INTRODUCTION}

The cardiovascular community has been confused about medical management for patients with peripheral artery disease (PAD) undergoing endovascular therapy. It is widely recognised that antiplatelet therapy is an integral part of preventing cardiovascular mortality and morbidity in patients with PAD. ${ }^{1}$ The femoropopliteal artery is the most common site of involvement in patients with atherosclerotic PAD. ${ }^{2}$ With technological improvements in the endovascular armamentarium, nitinol stenting could be one of treatment options for femoropopliteal artery disease. ${ }^{34}$ Given the high chance

\section{Key messages}

What is already known about this subject?

- The femoropopliteal artery is the most common site of involvement in patients with atherosclerotic peripheral artery disease.

- Nitinol stenting has become the standard of care in the treatment of femoropopliteal artery disease.

- An appropriate antithrombotic therapy after femoropopliteal intervention remains unclear.

What does this study add?

- Freedoms from target lesion revascularisation (TLR) and major adverse cardiovascular and cerebrovascular events (MACCE) at 12 months after femoropopliteal stenting with secondgeneration nitinol bare metal stent were $89.4 \%$ and $89.7 \%$, respectively.

- Neither dual antiplatelet therapy nor cilostazol at discharge was associated with freedom from TLR and MACCE at 12 months.

- Warfarin at discharge was independently associated with TLR at 12 months, and the 12 month Kaplan-Meier estimates found a significantly lower freedom from TLR in warfarin at discharge than no warfarin at discharge.

How might this impact on clinical practice?

- Further studies regarding antiplatelet and anticoagulation therapies in the real-world practice would provide insights into an appropriate antithrombotic therapy after femoropopliteal intervention.

of restenosis and need for further intervention after femoropopliteal intervention, a couple of recent studies have addressed the effects of dual antiplatelet therapy (DAPT), defined the use of a P2Y12 receptor inhibitor and aspirin, and cilostazol in reducing restenosis and repeat revascularisation..$^{5-8}$ However, few reports are available regarding the effects of antithrombotic therapy after femoropopliteal intervention in the real-world practice. ${ }^{9}$

The aim of this study was to investigate the effects of antithrombotic therapy on clinical outcomes such as target lesion revascularisation (TLR) and major adverse cardiovascular and cerebrovascular events (MACCEs) at 12 months after femoropopliteal intervention with 
second-generation bare metal nitinol stents based on results from the Japan multicentre postmarketing surveillance.

\section{METHODS}

\section{Population}

The second-generation bare metal nitinol stent, Misago stent (Terumo Corp, Tokyo, Japan) Japan postmarketing surveillance was performed to evaluate the safety and efficacy of the Misago stent in routine daily practice in the Japanese population. A total of 295 patients who underwent Misago stent deployment were registered between January 2013 and February at 78 sites in Japan. Of these, 277 lesions in 258 limbs of 248 patients with de novo atherosclerosis in the abovethe-knee femoropopliteal segment were evaluated. At each participating institution, patients were treated according to the local strategy. The regimen of drugs including antithrombotic agents was decided by the attending physician. TLR was also performed based on the attending physician's decision. Annual clinical follow-up was scheduled for up to 5 years. The informed consent for the postmarketing surveillance was given by all patients, and the institutional review boards approved the study.

\section{Endpoints}

The primary endpoint of this study was TLR at 12 months. The secondary endpoint was the composite outcome of MACCE at 12 months. TLR was defined as any repeat percutaneous intervention or bypass surgery of the target lesion performed for restenosis or reocclusion. MACCE was defined as myocardial infarction, ischaemic stroke and all-cause death. Major bleeding was defined as fatal bleeding.

\section{Statistical analysis}

Normally distributed continuous variables are presented as means $\pm S D$; they were compared using the t-test. Non-normally distributed variables were compared using the MannWhitney U test; categorical baseline variables were compared using Fisher's exact test, the $\chi^{2}$ test or analysis of variance as appropriate. Cumulative TLR rates and MACCE rates during the follow-up period were estimated using the Kaplan-Meier method and compared using the log-rank test. Cox proportional hazards model was used for univariate/multivariable analyses to assess the influence of factors on TLR and MACCE. Variables with a value of $\mathrm{p}<0.10$ at clinical characteristics analysis were entered into the regression model. Also, the shared-frailty Cox model was conducted with each institute and ID as a random intercept. All statistical analysis was performed with SPSS V.22 and STATA V.14. All $\mathrm{p}$ value $<0.05$ was considered statistically significant.

\section{RESULTS}

\section{Baseline characteristics}

Overall 248 patients, 258 limbs and 277 lesions characteristics are summarised in table 1 . The most prevalent risk factor was hypertension, followed by diabetes mellitus. The mean lesion length was $12.5 \pm 9.8 \mathrm{~cm}$, and $41.9 \%$ of lesions had chronic total occlusion (CTO). The number of stents implanted was 1.6 \pm 0.9 .

At discharge, DAPT was prescribed for $68.5 \%$ (170) and cilostazol for $30.2 \%$ (75) of patients. Warfarin was prescribed $14.5 \%$ (36). The DAPT group had a significantly higher prevalence of previous coronary revascularisation and a significantly lower prevalence of cilostazol and severe calcified lesion than

\begin{tabular}{|c|c|}
\hline \multicolumn{2}{|l|}{ Patient characteristics } \\
\hline Patients, n & 248 \\
\hline Age, years & $72.8 \pm 9.6$ \\
\hline Male, \% & 74.2 \\
\hline Prior lower limb revascularisation, \% & 37.2 \\
\hline Prior coronary revascularisation, \% & 44.7 \\
\hline Hypertension, \% & 79.8 \\
\hline Diabetes mellitus, $\%$ & 62.0 \\
\hline Dyslipidaemia, \% & 57.2 \\
\hline Haemodialysis, \% & 29.0 \\
\hline Smoking history, \% & 67.8 \\
\hline \multicolumn{2}{|l|}{ Limb characteristics } \\
\hline Limbs, n & 258 \\
\hline Right, $\mathrm{n}$ & 131 \\
\hline \multicolumn{2}{|l|}{ Lesion characteristics } \\
\hline Lesions, $\mathrm{n}$ & 277 \\
\hline Reference vessel diameter, mm & $5.8 \pm 0.8$ \\
\hline Lesion length, mm & $124.9 \pm 97.6$ \\
\hline Chronic total occlusion, \% & 41.9 \\
\hline Severe calcification, \% & 16.2 \\
\hline \multicolumn{2}{|l|}{ Extent of lesion, \% } \\
\hline Proximal SFA & 23.1 \\
\hline Distal SFA & 38.6 \\
\hline Above-the-knee PA & 5.1 \\
\hline Entire SFA & 27.4 \\
\hline Distal SFA and above-the-knee PA & 2.2 \\
\hline Entire SFA and above-the-knee PA & 3.6 \\
\hline Number of stents used & $1.6 \pm 0.9$ \\
\hline
\end{tabular}

PA, popliteal artery; SFA, superficial femoral artery.

the no DAPT group. Warfarin showed a trend towards lower prevalence in the DAPT group than the no DAPT group. Also, there was a significantly higher prevalence of DAPT and previous coronary revascularisation in the no cilostazol group than in the cilostazol group (tables 2 and 3 ).

At 12 months of follow-up, prescription of DAPT significantly $(\mathrm{p}=0.0001)$ decreased to $51.2 \%$, whereas prescription of cilostazol remained significantly unchanged $(\mathrm{p}=0.592)$ at $28.0 \%$. Warfarin also remained significantly unchanged $(\mathrm{p}=0.70)$ at $13.3 \%$. The DAPT group still had a significantly higher prevalence of previous coronary and lower prevalence of cilostazol and severe calcified lesion than the no DAPT group (online supplementary table 1). Except for DAPT, there was no significant difference in clinical and lesion characteristics between the cilostazol and the no cilostazol groups (online supplementary table 2).

\section{Clinical outcomes}

At 12 months, the stent thrombosis rate on limb basis was $1.2 \%(3 / 258)$, and the stent fracture rate on lesion basis was $1.1 \%(3 / 277)$. Major bleeding (intracranial haemorrhage) was observed in $0.4 \%$ (1/248). Significant improvement in Rutherford classification $(p<0.0001)$ was observed (table 4$)$. Clinical improvement of at least 1 Rutherford classification was achieved in $85.4 \%$, and no significant difference was observed between treatment groups at discharge (DAPT+: $85.5 \%$ vs DAPT$85.2 \% ; \mathrm{p}=0.965$, cilostazol+: $84.8 \%$ vs $84.4 \% ; \mathrm{p}=0.947)$. Also, ankle-brachial index $(\mathrm{ABI})$ significantly $(\mathrm{p}<0.0001)$ improved at 12 months from $0.63 \pm 0.19$ to $0.84 \pm 0.18$. All treatment groups at discharge showed a significant $(\mathrm{p}<0.001)$ 
Table 2 Clinical characteristics based on medical therapy at discharge

\begin{tabular}{|c|c|c|c|c|c|c|}
\hline & DAPT $(+)(n=170)$ & DAPT $(-)(n=78)$ & $P$ value & $\begin{array}{l}\text { Cilostazol (+) } \\
(n=75)\end{array}$ & $\begin{array}{l}\text { Cilostazol (-) } \\
(n=173)\end{array}$ & $P$ value \\
\hline Age, years & $72.3 \pm 9.5$ & $73.9 \pm 9.6$ & 0.24 & $72.8 \pm 10.4$ & $72.8 \pm 9.2$ & 0.99 \\
\hline Male sex, \% & 77.6 & 66.7 & 0.067 & 73.3 & 74.6 & 0.84 \\
\hline Prior lower limb revascularisation, $\%$ & 40.8 & 29.5 & 0.087 & 35.1 & 38.2 & 0.65 \\
\hline Prior coronary revascularisation, $\%$ & 55 & 22.1 & $<0.001$ & 26.7 & 52.6 & $<0.001$ \\
\hline Hypertension, \% & 79.6 & 80.3 & 0.91 & 79.7 & 79.9 & 0.98 \\
\hline Diabetes mellitus, $\%$ & 61.3 & 63.6 & 0.73 & 64.9 & 60.8 & 0.55 \\
\hline Dyslipidaemia, \% & 60.5 & 50 & 0.13 & 52.7 & 59.2 & 0.35 \\
\hline Haemodialysis, \% & 29.8 & 27.3 & 0.69 & 23 & 31.6 & 0.17 \\
\hline Smoking history, \% & 70.5 & 61 & 0.19 & 64.4 & 69.2 & 0.51 \\
\hline DAPT administration, $\%$ & & & & 40 & 80.9 & $<0.001$ \\
\hline Cilostazol administration, \% & 17.6 & 57.7 & $<0.001$ & & & \\
\hline Warfarin administration, $\%$ & 11.8 & 20.5 & 0.069 & 10.7 & 16.2 & 0.26 \\
\hline
\end{tabular}

DAPT, dual antiplatelet therapy.

improvement of $\mathrm{ABI}$ (DAPT+: $0.63 \pm 0.19$ to $0.85 \pm 0.18$, DAPT $-: 0.62 \pm 0.20$ to $0.83 \pm 0.16$, cilostazol $+: 0.60 \pm 0.20$ to $0.82 \pm 0.17$, cilostazol $-: 0.64 \pm 0.19$ to $0.85 \pm 0.18$ ), and no significant difference in change of ABI was observed between treatment groups at discharge (DAPT + vs DAPT $-; p=0.848$, cilostazol+ vs cilostazol-; $\mathrm{p}=0.685)$. No major amputation was observed, while minor amputation was executed in two limbs of one patient receiving warfarin in no DAPT and no cilostazol groups at discharge. Therefore, minor amputation rate was $0.4 \%(1 / 248)$ on patient basis and $0.8 \%(2 / 258)$ on limb basis.

\section{TLR at 12 months}

Kaplan-Meier estimates showed that freedom from TLR was $89.4 \%$ (95\% CI 84.7 to 92.7 ) at 12 months (figure $1 \mathrm{~A}$ ) and significantly $(p=0.044)$ higher freedom from TLR in DAPT group at discharge than no-DAPT group at discharge (figure 1B). However, there was no significant $(p=0.892)$ difference in freedom from TLR between the cilostazol and no cilostazol groups at discharge (figure 1C). The multivariate Cox proportional hazards model identified warfarin at discharge as the only independent predictor of TLR (table 5). According to Kaplan-Meier estimates, freedom from TLR was significantly $(p=0.013)$ lower in warfarin group at discharge than no warfarin group at discharge (figure 1D). Freedom from TLR at 12 months by the Kaplan-Meier estimates was
$77.8 \%$ (95\% CI $59.0 \%$ to $88.8 \%$ ) in patients with warfarin at discharge and $91.2 \%$ (95\% CI $86.3 \%$ to $94.3 \%)$ in those without warfarin at discharge.

\section{MACCEs at 12 months}

Kaplan-Meier estimates showed that freedom from MACCE was $89.7 \%$ (95\% CIs 85 to 93 ) at 12 months (figure 2A) and found no significant difference in freedom from MACCE between the DAPT and no DAPT groups at discharge $(p=0.268)$ and the cilostazol and no cilostazol groups at discharge $(p=0.268)$, respectively (figure $2 B$ and $C)$. The multivariate Cox proportional hazards model found no independent predictor of MACCE (table 6).

\section{DISCUSSION}

The main findings of this study were as follows: (1) the 12-month Kaplan-Meier estimates of freedom from TLR and MACCE were $89.4 \%$ and $89.7 \%$, respectively; $(2)$ in the multivariate Cox proportional hazards model, neither DAPT nor cilostazol at discharge was associated with both TLR and MACCE at 12 months, and warfarin at discharge was only independently associated with TLR at 12 months; and (3) the 12-month Kaplan-Meier estimates demonstrated a significantly lower freedom from TLR in warfarin at discharge than no warfarin at discharge.

Table 3 Lesion characteristics based on medical therapy at discharge

\begin{tabular}{|c|c|c|c|c|c|c|}
\hline & DAPT $(+)(n=189)$ & DAPT $(-)(n=88)$ & $P$ value & $\begin{array}{l}\text { Cilostazol (+) } \\
(n=84)\end{array}$ & $\begin{array}{l}\text { Cilostazol (-) } \\
(n=193)\end{array}$ & $P$ value \\
\hline Reference vessel diameter, mm & $5.8 \pm 0.9$ & $5.7 \pm 0.7$ & 0.193 & $5.7 \pm 0.8$ & $5.8 \pm 0.9$ & 0.149 \\
\hline Lesion length, mm & $129.0 \pm 98.7$ & $116.1 \pm 95.1$ & 0.307 & $136.4 \pm 99.8$ & $119.9 \pm 96.4$ & 0.198 \\
\hline Chronic total occlusion, $\%$ & 42.8 & 42.9 & 0.991 & 48.7 & 40.4 & 0.211 \\
\hline Severe calcification, \% & 14.3 & 20.5 & 0.014 & 20.2 & 14.5 & 0.542 \\
\hline \multicolumn{7}{|l|}{ Extent of lesion, $\%$} \\
\hline Proximal SFA & 23.8 & 21.6 & 0.309 & 16.7 & 25.9 & 0.556 \\
\hline Distal SFA & 34.9 & 46.6 & & 42.9 & 36.8 & \\
\hline Above-the-knee PA & 4.2 & 6.8 & & 3.6 & 5.7 & \\
\hline Entire SFA & 30.7 & 20.5 & & 29.8 & 26.4 & \\
\hline Distal SFA and above-the-knee PA & 2.1 & 2.3 & & 2.4 & 2.1 & \\
\hline Entire SFA and above-the-knee PA & 4.2 & 2.3 & & 4.8 & 3.1 & \\
\hline Number of stents used & $1.6 \pm 0.9$ & $1.6 \pm 0.9$ & 0.875 & $1.7 \pm 0.9$ & $1.6 \pm 0.9$ & 0.294 \\
\hline
\end{tabular}

PA, popliteal artery; SFA, superficial femoral artery. 


\section{Original research}

Table 4 Clinical symptoms before and after revascularisation

\begin{tabular}{|c|c|c|c|c|c|c|c|}
\hline & Baseline & NA & At discharge & NA & At 12 months & NA & $P$ value \\
\hline Rutherford class, n (\%) & 231 & 27 & 213 & 45 & 170 & 88 & $<0.0001$ \\
\hline 0 & $3(1.3)$ & & $114(53.5)$ & & $95(55.9)$ & & \\
\hline 2 & $62(26.8)$ & & $14(6.6)$ & & $18(10.6)$ & & \\
\hline 3 & 79 (34.2) & & $12(5.6)$ & & $10(5.9)$ & & \\
\hline 4 & $32(13.9)$ & & $4(1.9)$ & & $6(3.5)$ & & \\
\hline
\end{tabular}

NA, not available.

With a robust popularity of endovascular intervention for symptomatic PAD, the number of femoropopliteal artery stenting procedures is increasing. ${ }^{10}$ Given the lack of homogeneity in definitions of in-stent restenosis across clinical studies of femoropopliteal stenting, ${ }^{11}$ TLR is a more practical metric than restenosis; it reflects the correlation between endovascular therapy and clinical outcomes. ${ }^{12} 13$ Previous clinical trials with current bare metal nitinol stents have reported that the TLR rate at 12 months ranges from $10.1 \%$ to $20.9 \%$ (mean lesion length: $6.4-11.2 \mathrm{~cm}$, CTO in $17 \%-64 \%$ of lesions). ${ }^{14-23}$
In these clinical trials, there is a broad range of minimum duration of antiplatelet therapy (1-3 months for clopidogrel or ticlopidine, indefinite to lifelong for aspirin). Even in the recent postmarketing survey with drug-eluting stent of Zilver PTX (Cook Medical Technologies LLC), which continued clopidogrel or ticlopidine for at least 60-day postprocedure and aspirin indefinitely, freedom from TLR at 12 months was $91 \% .{ }^{24}$ In the present study in which patient characteristics (diabetes in 62\% and haemodialysis in 29\%) and lesion characteristics (mean lesion length of $12.5 \mathrm{~cm}$, CTO in $41.9 \%$ and
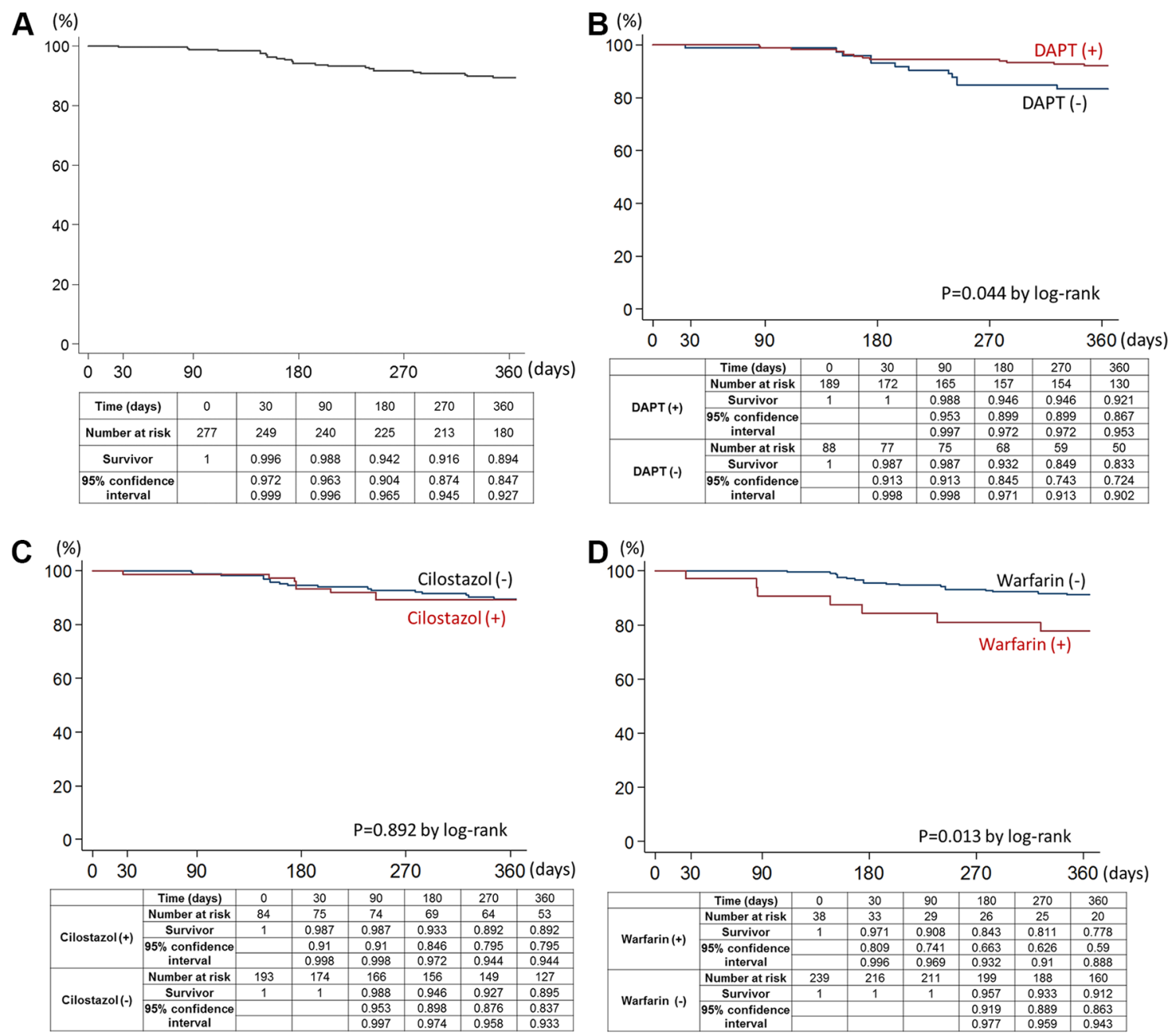

Figure 1 (A) Kaplan-Meier curve at 12 months of follow-up for freedom from target lesion revascularisation. (B) Kaplan-Meier curves at 12 months of follow-up for freedom from target lesion revascularisation by DAPT status at discharge. (C) Kaplan-Meier curves at 12 months of follow-up for freedom from target lesion revascularisation by cilostazol status at discharge. (D) Kaplan-Meier curves at 12 months of follow-up for freedom from target lesion revascularisation by warfarin status at discharge. DAPT, dual antiplatelet therapy. 
Table 5 Cox proportional hazards model for TLR at 12 months

\begin{tabular}{|c|c|c|c|c|c|c|c|c|c|}
\hline & \multicolumn{3}{|c|}{ Univariate } & \multicolumn{3}{|c|}{ Multivariate model 1} & \multicolumn{3}{|c|}{ Multivariate model 2} \\
\hline & HR & $95 \% \mathrm{Cl}$ & $P$ value & HR & $95 \% \mathrm{Cl}$ & $P$ value & HR & $95 \% \mathrm{Cl}$ & P value \\
\hline DAPT at discharge & 0.46 & (0.21 to 1.00$)$ & 0.05 & 0.43 & (0.17 to 1.08$)$ & 0.074 & 0.24 & (0.02 to 3.52$)$ & 0.297 \\
\hline Cilostazol at discharge & 1.06 & (0.46 to 2.46$)$ & 0.892 & 0.93 & (0.35 to 2.46$)$ & 0.889 & 0.29 & (0.02 to 3.62$)$ & 0.335 \\
\hline Warfarin at discharge & 2.87 & (1.20 to 6.86$)$ & 0.018 & 2.28 & (0.90 to 5.79$)$ & 0.082 & 31.70 & (1.46 to 685.89$)$ & 0.028 \\
\hline Male & 0.85 & (0.35 to 2.02 ) & 0.706 & 0.93 & (0.38 to 2.24$)$ & 0.869 & 0.88 & (0.12 to 6.60$)$ & 0.898 \\
\hline Prior lower limb revascularisation & 1.2 & (0.54 to 2.67 ) & 0.654 & 1.24 & (0.55 to 2.80$)$ & 0.602 & 2.44 & (0.31 to 19.50$)$ & 0.399 \\
\hline Prior coronary revascularisation & 1.14 & (0.52 to 2.50$)$ & 0.745 & 1.44 & (0.61 to 3.38$)$ & 0.407 & 1.64 & (0.23 to 11.85$)$ & 0.622 \\
\hline Severe calcified lesion & 0.69 & (0.45 to 1.07$)$ & 0.095 & 0.68 & (0.44 to 1.05$)$ & 0.084 & 0.44 & (0.16 to 1.23$)$ & 0.118 \\
\hline
\end{tabular}

Model 1: adjusted for DAPT at discharge, cilostazol at discharge, warfarin at discharge, male, prior lower limb revascularisation, prior coronary revascularisation and severe calcified lesion. Model 2: shared-frailty model with each institution and ID as a random intercept, adjusted for variable in model 1.

DAPT, dual antiplatelet therapy; TLR, target lesion revascularisation.

severe calcification in $16.2 \%$ ) could reflect the real world, freedom from TLR at 12 months was $89.4 \%$ with a significant improvement of ABI and Rutherford category. Also, the present study found no safety concerns. Although caution needs to be taken comparing outcomes between different studies, this second-generation nitinol bare metal stent appears to be acceptable in terms of safety and effectiveness. The administration of DAPT was in $68.5 \%$ at discharge and significantly decreased to $51.2 \%$ at 12 months but still remained high. Given that prior coronary revascularisation was observed in
A $(\%)$

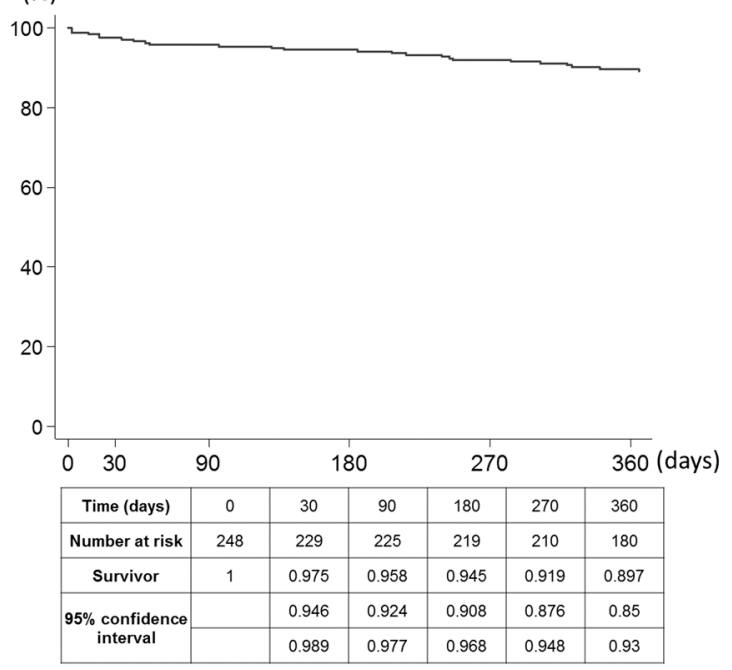

B $\%$ )

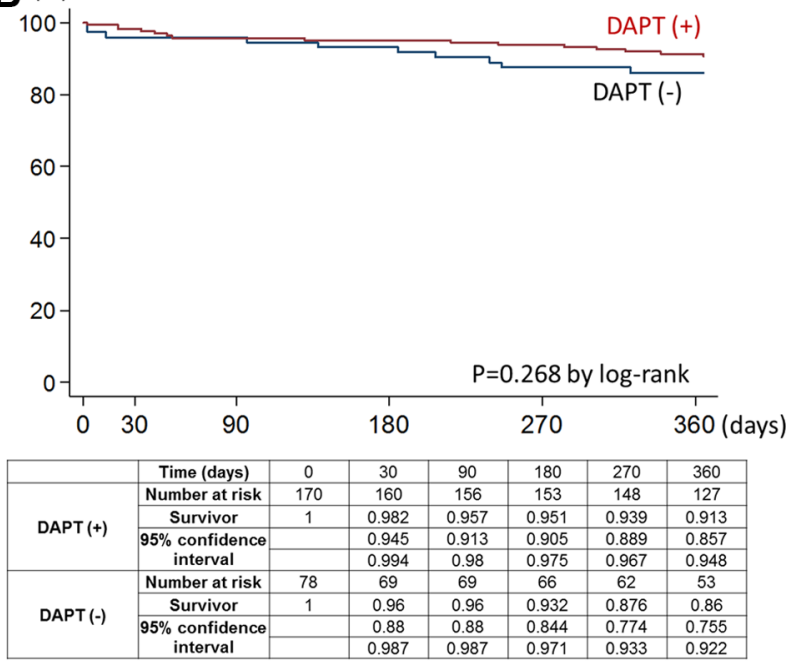

C (\%)

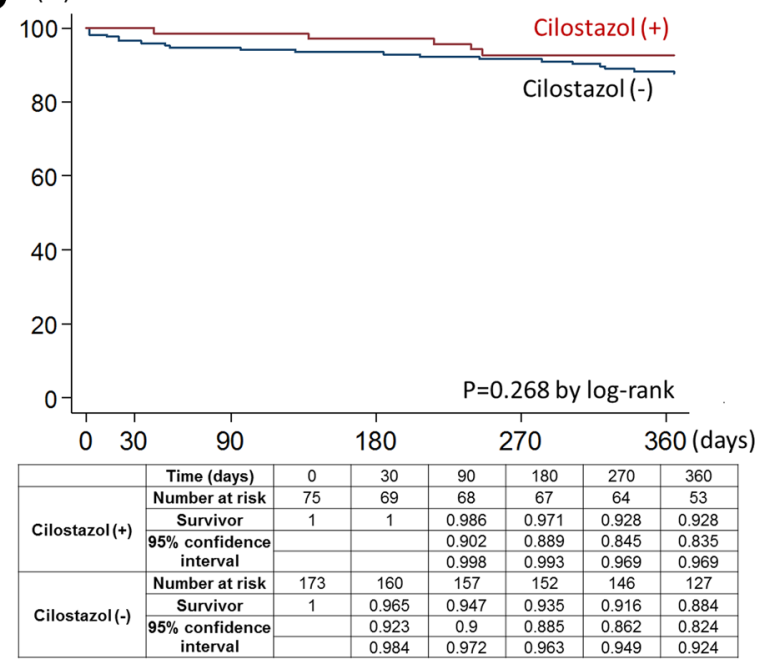

Figure 2 (A) Kaplan-Meier curve at 12 months of follow-up for freedom from major adverse cardiovascular and cerebrovascular events. (B) KaplanMeier curves at 12 months of follow-up for freedom from major adverse cardiovascular and cerebrovascular events by DAPT status at discharge. (C) Kaplan-Meier curves at 12 months of follow-up for freedom from major adverse cardiovascular and cerebrovascular events by cilostazol status at discharge. DAPT, dual antiplatelet therapy. 
Table 6 Cox proportional hazards model for MACCE at 12 months

\begin{tabular}{|c|c|c|c|c|c|c|c|c|c|}
\hline & \multicolumn{3}{|c|}{ Univariate } & \multicolumn{3}{|c|}{ Multivariate: model 1} & \multicolumn{3}{|c|}{ Multivariate: model 2} \\
\hline & HR & $95 \% \mathrm{Cl}$ & $P$ value & $H R$ & $95 \% \mathrm{Cl}$ & $P$ value & HR & $95 \% \mathrm{Cl}$ & $P$ value \\
\hline DAPT at discharge & 0.64 & (0.29 to 1.42$)$ & 0.272 & 0.41 & (0.15 to 1.13 ) & 0.086 & 0.41 & (0.15 to 1.13$)$ & 0.086 \\
\hline Cilostazol at discharge & 0.58 & (0.22 to 1.54$)$ & 0.274 & 0.48 & (0.16 to 1.46$)$ & 0.198 & 0.48 & (0.16 to 1.46$)$ & 0.198 \\
\hline Warfarin at discharge & 1.13 & (0.39 to 3.30$)$ & 0.821 & 0.89 & (0.28 to 2.88 ) & 0.851 & 0.89 & (0.28 to 2.88$)$ & 0.851 \\
\hline Male & 0.93 & (0.39 to 2.22 ) & 0.867 & 1.29 & (0.48 to 3.51$)$ & 0.615 & 1.29 & (0.48 to 3.51$)$ & 0.615 \\
\hline Prior lower limb revascularisation & 1.55 & (0.71 to 3.39 ) & 0.275 & 1.80 & (0.79 to 4.13$)$ & 0.163 & 1.8 & (0.79 to 4.13$)$ & 0.163 \\
\hline Prior coronary revascularisation & 1.32 & (0.58 to 2.99 ) & 0.506 & 1.37 & (0.56 to 3.36$)$ & 0.487 & 1.37 & (0.56 to 3.36$)$ & 0.487 \\
\hline
\end{tabular}

Model 1: adjusted for DAPT at discharge, cilostazol at discharge, warfarin at discharge, male, prior lower limb revascularisation and prior coronary revascularisation.

Model 2: shared-frailty model with each institution and ID as a random intercept, adjusted for variable in model 1.

DAPT, dual antiplatelet therapy; MACCE, major adverse cardiovascular and cerebrovascular event.

$55 \%$ and $61 \%$ at discharge and at 12 months in the DAPT group, the drive of DAPT administration might be extrapolated from clinical experience in coronary intervention. Also, reflecting no consensus on the role of cilostazol in the setting of postintervention, administration of cilostazol remained at approximately 30\% (30.2\% at discharge and $28.0 \%$ at 12 months) in the present study.

According to the MIRROR (Management of Peripheral Arterial Interventions with Mono or Dual Antiplatelet Therapy) study, ${ }^{5}$ which was a prospective randomised study to investigate the effect of aspirin and clopidogrel following femoropopliteal angioplasty or stenting, TLR at 6 months was significantly less common in the DAPT group than in the aspirin monotherapy group (5\% in the clopidogrel group vs $20 \%$ in the placebo group, $\mathrm{p}=0.04)$. When clopidogrel and placebo were stopped after 6 months, patients of both groups remained on aspirin only. There was no longer a significant difference in TLR rates at 12 months $(25 \%$ in the clopidogrel group and $32 \%$ in the placebo group). However, it remains unclear whether the disappearance of a difference is due to the discontinuation of clopidogrel or not. Also, a few prospective studies reported that cilostazol reduced restenosis, repeat revascularisation and cardiovascular events following femoropopliteal balloon angioplasty or stenting. ${ }^{78}$ However, there are some criticisms that these studies have been small and had an open-label design; thus, they were only hypothesis generating. ${ }^{25}$

In the present study, there was no significant difference in improvement of Rutherford category and improvement of ABI between patients with and without DAPT at discharge or patients with and without cilostazol at discharge. KaplanMeier estimates showed a significantly higher freedom from TLR within 12 months in DAPT group at discharge than no DAPT group at discharge, while no significant difference in TLR was observed between cilostazol group and no cilostazol group. However, of great interest, multivariate Cox proportional hazard model found that not DAPT or cilostazol but warfarin at discharge was independently associated with TLR. Prevalence of warfarin remained unchanged between at discharge and at 12 months $(14.5 \%$ and $13.3 \%, p=0.70)$, and Kaplan-Meier estimates demonstrated significantly lower freedom from TLR in warfarin at discharge than no warfarin at discharge. In the literature, there have been limited data regarding the role of warfarin, with and without antiplatelet therapy, in patients with PAD especially undergoing revascularisation. In the Dutch Bypass, Oral anticoagulants or Aspirin study, a multicentre, open, randomised trial, to compare the effectiveness of oral anticoagulants (phenprocoumon or acenocoumarol) such vitamin $\mathrm{K}$ antagonist with that of aspirin in the prevention of infrainguinal bypass graft occlusion and other clinical events, ${ }^{26}$ oral anticoagulants such as vitamin $\mathrm{K}$ antagonist yielded significantly higher prosthetic graft occlusion compared with aspirin. The risk of bleeding was almost doubled in the oral anticoagulation group, which may affect the original anti-thrombotic regimens. Furthermore, recent studies reported that warfarin was significantly associated with lower extremity arterial calcification. ${ }^{27} 28$ Therefore, this adverse effect of warfarin on the peripheral artery might one of the reasons for an increasing need for TLR after femoropopliteal revascularisation. Given the recently reported benefits of direct oral anticoagulants in symptomatic patients with PAD from multicentre double-blind randomised study, ${ }^{29}$ the clinical effects of warfarin on the peripheral artery might be distinct from those of direct oral anticoagulants.

Antiplatelet therapy is an integral part of preventing cardiovascular morbidity and mortality. According to updated guidelines, ${ }^{1} 30$ prescribing aspirin or clopidogrel in patients with symptomatic PAD has a class 1 recommendation with evidence level A. Furthermore, DAPT (ie, the combination of aspirin and clopidogrel) has a class IIb recommendation with evidence level B. In the present study, $68.5 \%$ received DAPT at discharge and $51.2 \%$ even at 12 months in parallel with previous coronary revascularisation. However, we found no association between DAPT at discharge and MACCE. Also, we found no other factors associated with MACCE. Therefore, the present study suggests that antiplatelet monotherapy might be reasonable even after femoropopliteal revascularisation in terms of prevention of cardiovascular mortality and morbidity, supporting the updated guidelines. $^{130}$

The present study has several potential limitations. First, our data are non-randomised and observational in nature and may therefore be affected by confounding. Given the patients' clinical background, administration of antithrombotic agents was determined by the attending physicians probably considering the risk and benefits trade-off in the real-world practice. Second, administration of antithrombotic agents was assessed at two time points such as at discharge and at 12 months, and no data are available regarding interruption and resumption of antithrombotic therapy within 12 months. However, administration of DAPT at 12 months still remained high at over 50\%, and approximately 30\% (30.2\% at discharge and $28.0 \%$ at 12 months) received cilostazol and approximately $15 \%(14.5 \%$ at discharge and $13.3 \%$ at 12 months) received warfarin during the study period. Therefore, the effects of 'on' and 'off' medication fluctuation could be considered small. Finally, the dose of aspirin and cilostazol and the intensity of warfarin might affect the clinical outcomes. Also, although it is speculated that most of patients with warfarin had standard indications such as atrial 
fibrillation, valve replacement and deep vein thrombosis for anticoagulation, the reason for warfarin administration is not reported in this postmarketing surveillance.

\section{CONCLUSION}

In the real world practice, clinical benefits of DAPT or cilostazol might be small in terms of TLR and MACCE at 12 months. Anticoagulation with warfarin at discharge might increase TLR at 12 months.

\section{Author affiliations \\ 'Department of Cardiovascular Medicine, National Cerebral and Cardiovascular Center, Osaka, Japan \\ ${ }^{2}$ Department of Statistics and Data Analysis, Center for Cerebral and Cardiovascular Disease Information, National Cerebral and Cardiovascular Center, Osaka, Japan \\ ${ }^{3}$ Clinical Development Department, Terumo Corporation, Tokyo, Japan \\ ${ }^{4}$ Division of Cardiology, Osaka General Medical Center, Osaka, Japan \\ ${ }^{5}$ Cardiovascular Medicine, Sapporo Cardiovascular Clinic, Hokkaido, Japan \\ ${ }^{6}$ Department of Cardiology, Miyazaki Medical Association Hospital, Miyazaki, Japan \\ 'Department of Cardiology, Iwaki Kyoritsu General Hospital, Fukushima, Japan \\ ${ }^{8}$ Department of Cardiology, Ageo Central General Hospital, Saitama, Japan \\ ${ }^{9}$ Division of Cardiovascular Medicine, Toho University Ohashi Medical Center, Tokyo, Japan}

Acknowledgements We would like to thank the investigators and institutions that participated in the Misago stent Japan postmarketing surveillance.

Contributors The contribution of each author is as follow: (1) conception and design or analysis and interpretation of data, or both: OK, MN and KN; (2) drafting of the manuscript or revising it critically for important intellectual content: OK, NO, MN and SY and (3) data collection: OK, HM, YI, DK, TN and YY.

Funding The authors have not declared a specific grant for this research from any funding agency in the public, commercial or not-for-profit sectors.

Competing interests OK reports honorarium of lectures and advisory board fees from Boston Scientific Corporation, honorarium of lectures and research grants from Terumo, and a consultancy fee from Medtronic. MN and KN report consigned research funds from Terumo. TN reports honorarium of lectures from Abbott Vascular, Boston Scientific and Medtronic and consulting fee from Boston Scientific and Century Medical Inc

Patient consent Not required.

Ethics approval National Cerebral and Cardiovascular Center.

Open access This is an open access article distributed in accordance with the Creative Commons Attribution Non Commercial (CC BY-NC 4.0) license, which permits others to distribute, remix, adapt, build upon this work non-commercially, and license their derivative works on different terms, provided the original work is properly cited, appropriate credit is given, any changes made indicated, and the use is non-commercial. See: http://creativecommons.org/licenses/by-nc/4.0/.

\section{REFERENCES}

1 Gerhard-Herman MD, Gornik HL, Barrett C, et al. 2016 AHA/ACC guideline on the management of patients with lower extremity peripheral artery disease: a report of the american college of cardiology/american heart association task force on clinical practice guidelines. Circulation 2017;135:e726-e779.

2 Kasapis C, Gurm HS. Current approach to the diagnosis and treatment of femoralpopliteal arterial disease. A systematic review. Curr Cardiol Rev 2009;5:296-311.

3 Feldman DN, Armstrong EJ, Aronow HD, et al. SCAI consensus guidelines for device selection in femoral-popliteal arterial interventions. Catheter Cardiovasc Interv 2018.

4 Yokoi Y. How should recent endovascular trials for femoropopliteal artery disease be interpreted? Cardiovasc Interv Ther 2017:32:106-13.

5 Tepe G, Bantleon R, Brechtel K, et al. Management of peripheral arterial interventions with mono or dual antiplatelet therapy--the MIRROR study: a randomised and double-blinded clinical trial. Eur Radiol 2012;22:1998-2006.

6 Strobl FF, Brechtel K, Schmehl J, et al. Twelve-month results of a randomized trial comparing mono with dual antiplatelet therapy in endovascularly treated patients with peripheral artery disease. J Endovasc Ther 2013;20:699-706.

7 lida O, Yokoi H, Soga Y, et al. Cilostazol reduces angiographic restenosis after endovascular therapy for femoropopliteal lesions in the Sufficient Treatment of Peripheral Intervention by Cilostazol study. Circulation 2013;127:2307-15.
8 Soga Y, Yokoi H, Kawasaki T, et al. Efficacy of cilostazol after endovascular therapy for femoropopliteal artery disease in patients with intermittent claudication. J Am Coll Cardiol 2009;53:48-53.

9 Hess CN, Norgren L, Ansel GM, et al. A structured review of antithrombotic therapy in peripheral artery disease with a focus on revascularization: a tasc (intersociety consensus for the management of peripheral artery disease) initiative. (irculation 2017; 135:2534-55.

10 Hertzer NR. The natural history of peripheral vascular disease. Implications for its management. Circulation 1991;83(2 Suppl):112-19.

11 Higashimori A, Kawarada O, Morioka N, et al. Impact of changing PSVR thresholds on the patency rates of SFA recanalisation with self-expanding nitinol stents. EuroIntervention 2013;9:964-7.

12 Rosenschein U, Topol EJ. Uncoupling clinical outcomes and coronary angiography: a review and perspective of recent trials in coronary artery disease. Am Heart $J$ 1996:132:910-20

13 Mwipatayi $\mathrm{BP}$, Leong $\mathrm{BD}$, Hockley J, et al. The pitfalls of femoropopliteal stenting trials. $J$ Endovasc Ther 2012:19:596-8.

14 Bosiers M, Torsello G, Gissler HM, et al. Nitinol stent implantation in long superficial femoral artery lesions: 12-month results of the DURABILITY I study. J Endovasc Ther 2009;16:261-9.

15 Matsumura JS, Yamanouchi D, Goldstein JA, et al. The United States study for evaluating endovascular treatments of lesions in the superficial femoral artery and proximal popliteal by using the protégé everflex nitinol stent system II (DURABILITY II). J Vasc Surg 2013:58:73-83.

16 Laird JR, Katzen BT, Scheinert D, et al. Nitinol stent implantation versus balloon angioplasty for lesions in the superficial femoral artery and proximal popliteal artery: twelve-month results from the RESILIENT randomized trial. Circ Cardiovasc Interv 2010:3:267-76.

17 Garcia L, Jaff MR, Metzger C, et al. Wire-interwoven nitinol stent outcome in the superficial femoral and proximal popliteal arteries: twelve-month results of the SUPERB Trial. Circ Cardiovasc Interv 2015;8:e000937.

18 Krankenberg H, Tübler T, Sixt S, et al. German multicenter real-world registry of stenting for superficial femoral artery disease: clinical results and predictive factors for revascularization. J Endovasc Ther 2014;21:463-71.

19 Bosiers M, Deloose K, Callaert J, et al. 4-French-compatible endovascular material is safe and effective in the treatment of femoropopliteal occlusive disease: results of the 4-EVER trial. J Endovasc Ther 2013:20:746-56.

20 Lichtenberg M, Kolks O, Hailer B, et al. PEACE I all-comers registry: patency evaluation after implantation of the 4-French Pulsar-18 self-expanding nitinol stent in femoropopliteal lesions. J Endovasc Ther 2014;21:373-80.

21 Laird JR, Jain A, Zeller T, et al. Nitinol stent implantation in the superficial femoral artery and proximal popliteal artery: twelve-month results from the complete SE multicenter trial. J Endovasc Ther 2014:21:202-12.

22 Schulte KL, Kralj I, Gissler HM, et al. MISAGO 2: one-year outcomes after implantation of the Misago self-expanding nitinol stent in the superficial femoral and popliteal arteries of 744 patients. J Endovasc The 2012:19:774-84

23 Ohki T, Angle JF, Yokoi H, et al. One-year outcomes of the U.S. and Japanese regulatory trial of the Misago stent for treatment of superficial femoral artery disease (OSPREY study). J Vasc Surg 2016;63:370-6.

24 Yokoi H, Ohki T, Kichikawa K, et al. Zilver PTX post-market surveillance study of paclitaxel-eluting stents for treating femoropopliteal Artery Disease in Japan: 12-Month Results. JACC Cardiovasc Interv 2016;9:271-7.

25 Berger JS, Hiatt WR. Medical therapy in peripheral artery disease. Circulation 2012;126:491-500.

26 The Lancet. Efficacy of oral anticoagulants compared with aspirin after infrainguinal bypass surgery (The Dutch Bypass Oral anticoagulants or Aspirin study): a randomised trial. The Lancet 2000:355:346-51.

$27 \mathrm{Han} \mathrm{KH}, \mathrm{O}^{\prime}$ Neill WC. Increased peripheral arterial calcification in patients receiving warfarin. J Am Heart Assoc 2016:5:pii: e002665.

28 Poterucha TJ, Goldhaber SZ. Warfarin and Vascular Calcification. Am J Med 2016;129:635.e1-635.e4.

29 Anand SS, Bosch J, Eikelboom JW, et al. Rivaroxaban with or without aspirin in patients with stable peripheral or carotid artery disease: an international, randomised, double-blind, placebo-controlled trial. Lancet 2018;391:219-29.

30 Aboyans V, Ricco JB, Bartelink MEL, et al. 2017 ESC Guidelines on the Diagnosis and Treatment of Peripheral Arterial Diseases, in collaboration with the European Society for Vascular Surgery (ESVS): Document covering atherosclerotic disease of extracranial carotid and vertebral, mesenteric, renal, upper and lower extremity arteriesEndorsed by: the European Stroke Organization (ESO)The Task Force for the Diagnosis and Treatment of Peripheral Arterial Diseases of the European Society of Cardiology (ESC) and of the European Society for Vascular Surgery (ESVS). Eur Heart $J$ 2018:39:763-816. 